\title{
A pós-graduação em educação no Brasil e no Canadá: aproximações e distanciamentos nos critérios de avaliação
}

\author{
Isabela Cristina Marins Braga, Ranilce \\ Guimarães-Iosif \& Lynette Shultz
}

Resumo:

O presente artigo tem o objetivo de compreender o modelo de pós- graduação em educação adotado no Brasil e no Canadá, com o intuito de tecer uma análise sobre as aproximações e os distanciamentos dos critérios de avaliação presentes nesses diferentes contextos. O método utilizado se aproxima de uma análise comparativa e utiliza a análise documental como técnica de pesquisa, tomando como referência os principais documentos que normatizam a pós-graduação nos dois países. Os documentos foram analisados a luz das seguintes categorias: organicidade política, avaliação do corpo docente e discente, produção intelectual e inserção social. De modo geral, a pós-graduação brasileira e canadense mostra-se alinhada com a mudança na cultura acadêmica que se fundamenta na avaliação e na performatividade das atividades desenvolvidas. A diferença se esbarra principalmente no aspecto da autonomia e de um maior equilíbrio entre critérios quantitativos e qualitativos, que são mais evidentes no Canadá. O estudo ressalta a necessidade de uma minuciosa análise sobre os desafios e perspectivas vivenciados pela pós-graduação nos contextos investigados, sobretudo no que se refere ao engajamento social e à prática da pesquisa que, dado à demanda por publicação, especialmente no caso brasileiro, pode ficar comprometida.

Palavras-chave:

pós-graduação; Brasil e Canadá; avaliação; pesquisa e prática social. 


\title{
The postgraduate in Education in Brazil and Canada: similarities and differ- ences in the evaluation criteria.
}

\begin{abstract}
The intent of this article is to understand the postgraduate model in education adopted in Brazil and Canada with the purpose of analyzing the similarities and differences of the evaluation criteria adopted in these different contexts. The method used is a comparative analysis and uses document analysis as a research technique, referencing the main documents that outline standards of the postgraduate in both countries. The documents were classified under the following categories: program structure and governance, evaluation of faculty and students, intellectual production/dissemination and social engagement. In general, Brazilian and Canadian postgraduate studies are aligned with shifts in academic culture that are reflected in the performance and evaluation of academic activities. Differences surface in areas related to autonomy and the balance between quantitative and qualitative criteria, which are more evident in Canada. The study emphasizes the need for a thorough analysis of the challenges experienced by and the perspectives of the graduate students in the contexts investigated, especially with regard to social engagement and research practice, given the demand for publication, in the Brazilian context and its potential to compromise social engagement.
\end{abstract}

Key words: Postgraduate; Brazil and Canada; evaluation; research and social practice.

\section{Le études de troisième cycle en éducation au Brésil et au Canada: approxima- tions et distances dans les critères d'évaluation.}

Résumé: Cet article vise à comprendre le modèle études de troisième cycle en éducation adopté au Brésil et au Canada, dans le but d'analyser les approximations et les distances des critères d'évaluation présents dans ces différents contextes. La méthode utilisée se rapproche d'une analyse comparative et utilise l'analyse documentaire comme technique de recherche, prenant comme référence les principaux documents qui standardisent le troisième cycle dans les deux pays. Les documents ont été analysés à la lumière des catégories suivantes: organicité politique, évaluation des professeurs et des étudiants, production intellectuelle et insertion sociale. En général, les programmes de troisième cycle brésilien et canadien sont alignés sur le changement dans la culture académique qui est basé sur l'évaluation et la performativité des activités développées. La différence réside principalement dans l'aspect de l'autonomie et dans un meilleur équilibre entre les critères quantitatifs et qualitatifs, qui sont plus évidents au Canada. L'étude souligne la nécessité d'une analyse approfondie des défis et perspectives des étudiants diplômés dans les contextes étudiés, notamment en ce qui concerne l'engagement social et la pratique de la recherche qui, étant donné la demande de publication, notamment dans le cas brésilien, être compromis.

Mots-clés: études de troisième cycle; Brésil et Canada; évaluation; recherche et pratique sociale.

\section{El postgrado en Educación en Brasil y Canadá: aproximaciones y distancia- mientos en los criterios de evaluación.}

Resumen: El presente artículo tiene el objetivo de comprender el modelo de postgrado en educación adoptado en Brasil y Canadá, con el fin de hacer un análisis sobre las aproximaciones y los distanciamientos de los criterios de evaluación presentes en esos diferentes contextos. El método utilizado se aproxima a un análisis comparativo y utiliza el análisis documental como técnica de investigación, tomando como referencia los principales documentos que normalizan el posgrado en los dos países. Los documentos fueron analizados a la luz de las siguientes categorías: organicidad política, evaluación del cuerpo docente y discente, producción intelectual y la inserción social. En general, el postgrado brasileño y canadiense se muestra alineado con el cambio en la cultura académica que se fundamenta en la evaluación y en la performatividad de las actividades desarrolladas. La diferencia se da principalmente en el aspecto de la autonomía y de un mayor equilibrio entre criterios cuantitativos y cualitativos, que son más evidentes en Canadá. El estudio resalta la necesidad de un minucioso análisis sobre los desafíos y perspectivas vivenciados por el postgrado en los contextos investigados, sobre todo en lo que se refiere al compromiso social ya la práctica de la investigación que, dado a la demanda por publicación, especialmente en el caso brasileño, estar comprometida.

Palabras clave: posgrado; Brasil y Canadá; evaluación; investigación y práctica social. 
A partir da década de 1990, o imperativo da globalização foi ávido em promover profundas mudanças, aproximar nações e transpor as barreiras fronteiriças de problemas e dilemas políticos, econômicos e sociais. As pressões locais e globais sobre a educação são cada vez maiores, o que compromete seu papel social e o compromisso com a promoção de uma cidadania democrática, emancipatória e efetivamente para todos (Viczko \& Tascón, 2016; Zheng, 2010).

Na pós-graduação, essa tendência pode ser ainda mais danosa porque é nesse espaço que se formam os futuros mestres e doutores, intelectuais públicos cuja formação e atuação deveria ser pensada a favor da promoção social (Bourdieu, 2004). Com o avanço do modelo hegemônico de governança, as políticas públicas educacionais foram reconfiguradas com o intuito de abrir mais espaço para as parcerias público-privadas e deram ao Estado uma nova função, ligada especialmente à regulação. Para Mailhot, Pelletier e Schaeffer (2007) esse movimento global incorpora a figura da avaliação com o discurso voltado para a busca da qualidade, mas fundamentado, muitas vezes, no produtivismo e na performatividade.

Motivados pelas reflexões iniciais, objetivamos identificar os critérios de avaliação priorizados na pós-graduação em educação no Brasil e no Canadá, apoiados no modelo de análise comparativa apresentada por Franco (2000) para refletir sobre as aproximações e os distanciamentos entre os dois modelos sem a pretensão de reprodução ou imposição.

No contexto brasileiro, a pós-graduação é norteada por critérios de credenciamento e avaliação que, apesar de contar com a participação dos pares, integra o Sistema Nacional de Pós-graduação (SNPG) instituído pela Coordenação de Aperfeiçoamento de Pessoal de Nível Superior (Capes), agência que coordena os programas de mestrado e doutorado stricto sensu de todas as áreas e instituições do país. De acordo com Severino (2011), o nível stricto sensu é reconhecido no âmbito nacional e internacional pela qualidade das pesquisas que produz e pela consolidação e expansão dos programas em razão da política de avaliação e acompanhamento que preconiza.

No Canadá, a responsabilidade quanto à pós-graduação é distribuída em níveis de autoridades diferentes. Jones e Oleksiyenko (2011) esclarecem que o governo federal conduz as prioridades de financiamento da pesquisa, no entanto delega para as dez províncias o papel de regulação e financiamento das universidades. Por sua vez, as províncias concedem autonomia para que cada instituição defina critérios locais de oferta, atuação e avaliação de seus programas.

Desse modo, a base referencial de estudo no contexto canadense é University of Alberta (UofA), localizada na província de Alberta. A escolha por esta Instituição de Ensino Superior (IES) se justifica pelas relações de trabalho e pesquisa vivenciados pelos autores deste artigo e por se tratar de uma instituição, cuja área de educação é destaque a nível local e global' ${ }^{1}$. As diretrizes da pós-graduação em educação da UofA se fundamentam na avaliação por pares, na participação da governança institucional, na internacionalização e na disseminação da pesquisa na comunidade. 
O texto está estruturado em quatro partes: a primeira parte trata do método do estudo; a segunda parte busca explicar a organicidade do sistema brasileiro de pós- graduação em educação; a terceira parte visa compreender como se estrutura o sistema canadense de pós-graduação em educação e, por fim, a quarta parte discute as aproximações e distanciamentos entre os dois modelos.

\section{O método do estudo}

O presente estudo se aproxima da comparação como método investigativo. De acordo com Franco (2000) fazer analogias ou comparações está presente no cotidiano das pessoas, ligado a própria natureza humana. Contudo, não se trata simplesmente de julgar elementos ou ações como melhores ou piores, mas compreender e reconhecer sua existência sem desconsiderar o processo histórico e cultural. Para dar corpo a esse método, o estudo utiliza a análise documental que, segundo Shiroma, Campos e Garcia (2005), contribui para decifrar conceitos, conteúdos e discursos presentes nos documentos que norteiam as políticas educacionais.

A principal lei que regulamenta o ensino superior na província de Alberta é a Post-Secondary Learning ACT (Alberta, 2003). Esta lei também estabelece o sistema de governança da UofA e serve de base para todos os procedimentos políticos adotados pela instituição. Desse modo, além da referida lei, foram analisados os seguintes documentos: Contrato de Docência (Faculty Agreement - University of Alberta, 2017a); Manual de Referência para Comitês de Avaliação do Corpo Docente (Reference Manual for Faculty Evaluation Committees - University of Alberta, 2017b) e Comitê de Avaliação do Corpo Docente da Faculdade de Educação (Faculty Evaluation Committee - Faculty of Education - University of Alberta, 2012).

No Brasil, os documentos investigados foram o Plano Nacional de Pós-Graduação (PNPG 2011-2020); o Documento de área referente à última avaliação quadrienal (2013-2016) e a ficha da última avaliação quadrienal da área (2013-2016). O estudo de tais documentos teve o cuidado de ser permeado por um sólido arcabouço teórico-metodológico, pois, conforme apontam Shiroma, Campos e Garcia (2005), a análise de documentos no campo das políticas públicas educacionais, empreende uma compreensão empírica, metodológica e teórica, de modo que as informações obtidas não passem de mera descrição textual, conforme explicam as autoras.

\section{A pós-graduação em educação: o sistema brasileiro}

De acordo com Boaventura (2009), a pós-graduação brasileira representa o ápice da formação educacional e envolve os segmentos stricto sensu que são de responsabilidade da Capes, que incluem o mestrado acadêmico e profissional e o doutorado; e 
lato sensu, que se refere à especialização orientada para o ensino e está vinculada ao Ministério da Educação (MEC). Os programas de pós-graduação estão distribuídos em IESs públicas e privadas.

Fundada pelo Decreto $n^{\circ}$ 29.741, de 11 de julho de 1951, a Capes passou por inúmeras mudanças que se destacaram especialmente pela demanda inicial voltada à formação de professores, para a lógica orientada pelas políticas reformistas da década de 1990, em prol da formação de pesquisadores. As atividades que desenvolve contemplam a evolução da política nacional de pós-graduação, cujas diretrizes foram ampliadas e modificadas ao longo dos seus planos. No atual PNPG (2011-2020), os objetivos se concentram no dinamismo da pós-graduação, internacionalização, formação de mestres e doutores para atender ao mercado acadêmico e não acadêmico e articulação com a educação básica (Brasil, 2010).

A grandiosidade do campo científico brasileiro pode ser vislumbrada nos três colégios e nove grandes áreas que possui, dentre as quais a Educação se destaca por ser uma das áreas mais bem-sucedidas em função da valiosa produção do conhecimento que desenvolve sobre inúmeros temas educacionais (Moreira, 2009). Atualmente, a área está representada por 172 programas, distribuídos entre mestrado e doutorado acadêmicos e mestrado profissional (Brasil, 2016).

De acordo com Oliveira, Amaral e Zaidan (2015) a pós-graduação em educação responde a questões fundamentais para o desenvolvimento do país. Dentre elas destacam-se: inserção social, com vistas a atender às demandas da educação básica; estudos voltados à perspectiva da internacionalização, dado o crescimento das publicações e intercâmbio de alunos e professores com outras instituições; aumento do número da produção intelectual e da qualificação dos periódicos da área e maior número de docentes qualificados nos diferentes níveis de ensino. As atividades desenvolvidas pela área são permeadas pela pesquisa, cujo acompanhamento é conduzido à luz da política de avaliação (Brasil, 2016).

A avaliação do SNPG, desde o ano de 1998, segue a orientação da Diretoria de Avaliação/Capes (DAV/Capes), realizada com a participação da comunidade acadêmico-científica representada pelos consultores ad hoc. Sua finalidade é assegurar e manter a qualidade dos cursos de mestrado e doutorado no país. Os referenciais para a avaliação são representados pelas fichas, relatórios e documentos de área.

A ficha de avaliação da área de educação do quadriênio 2013-2016 (Brasil, 2016) foi composta por cinco quesitos: 1) Proposta do programa; 2) Corpo docente; 3) Corpo discente, teses e dissertações; 4) Produção intelectual e, 5) Inserção social. Cada quesito apresenta um conjunto de dimensões e, com exceção do quesito Proposta do programa, cada dimensão é avaliada conforme um determinado peso.

Segundo o documento de área (Brasil, 2016), a referida quadrienal sofreu algumas modificações em relação à trienal de 2013. A principal alteração refere-se ao quesito 
4, quanto à produção intelectual, que passou a considerar a "média ponderada de até oito produções mais bem qualificadas por docente permanente", e não mais toda a produção intelectual como era nas demais avaliações. À propósito dessa alteração Oliveira, Amaral e Zaidan (2015) apresentaram uma discussão sobre a fragilidade das métricas vigentes e consideraram que o atual peso dado à produção intelectual decorre da falta de formulação de critérios que possam avaliar os demais quesitos com foco na qualidade.

Quanto ao corpo docente, os critérios de avaliação vislumbram a titulação e experiência do professor com as atividades que exerce no programa, o número de publicações, especialmente nos veículos mais bem qualificados, o número de alunos que orienta, o número de projetos de pesquisa que participa, o número de atividades de ensino que possui na graduação e as atividades de inserção acadêmica que realizada, tais como participação em eventos, comissões e comitês ad hoc (Brasil, 2016).

Para a avaliação discente, as exigências por produtividade (Brasil, 2016) destacam a necessidade de primar pela qualidade das teses e dissertações, tomando como referência a publicação dos resultados da pesquisa em veículos bem avaliados pela área; o período máximo de 4 anos para conclusão do doutorado e 2 anos para o mestrado, especialmente no caso dos bolsistas; participar de projetos de pesquisas, grupos de pesquisa e eventos.

Essa demanda de atividades pode se tornar mais árdua para os discentes, quando se analisa a questão do suporte financeiro. De acordo com o GEOCAPES², no ano de 2016 a CAPES distribuiu 100.433 bolsas de estudos e, desse total, apenas 3.271 foram para a área de Educação. Trata-se de um número muito pequeno, principalmente pelo fato de que as IESs públicas têm mais recursos disponíveis do que as privadas. Contudo, os alunos das instituições privadas, além de terem um acesso mais limitado às bolsas, precisam trabalhar e dar conta das atividades do programa.

Todos esses fatores convergem para uma contradição quanto ao quesito inserção social. Além de ter um peso menor na avaliação, este quesito fica comprometido em razão da demanda por produção intelectual, que pode ser agravado pelos impasses presentes no sistema, como por exemplo, a dificuldade de análises pormenorizadas e circunstanciadas, conforme destaca Barata (2017).

No caso da área de educação, a centralidade de uma avaliação padronizada dificulta uma análise qualitativa dos critérios e acaba comprometendo tanto o tempo para se pesquisar, quanto para divulgar e refletir sobre os resultados das pesquisas (Bourdieu, 2004). Notadamente, a avaliação protagonizada pela Capes é bem-sucedida e contribuiu para a consolidação da atual SNPG. Mas é premente estabelecer um diálogo sobre as vicissitudes da política de avaliação para que não se transforme apenas em um aparato regulatório do Estado. 


\section{A pós-graduação em educação: o sistema canadense}

No Canadá, a educação superior (higher education) tem seus fundamentos legislativos e regulatórios diferentes em cada província. O governo provincial custeia a maior parte das despesas das universidades públicas e o restante é cobrado da população através de taxas anuais. Há também a figura da doação de verbas para pesquisa por parte do setor privado, em razão da forte parceria entre universidade e indústria (Jones \& Oleksiyenko, 2011).

De acordo com a Post-Secondary Learning ACT (Alberta, 2003), a governança da University of Alberta é regida por um sistema bicameral, sendo o Conselho de Governadores responsável pela governança empresarial e o Conselho das Faculdades Gerais (GFC) responsável pela gestão acadêmica. Este estudo não pretende promover um debate exclusivo sobre as novas estratégias de gestão, mas é pertinente destacar o modelo de governança adotado pela University of Alberta.

Rhodes (1996) aponta que a governança simboliza um novo modo de governar que se compõe de novas práticas e regras de gestão e disciplina, que tem a sociedade como protagonista. Nesse modelo, o autor explica que o Estado concede maior autonomia aos atores envolvidos e se posiciona como um elemento central da regulação.

A gestão exercida na University of Alberta traz em sua concepção os pressupostos do atual modelo de governança educacional. Apesar de ter como pano de fundo a figura do Estado, a universidade possui autonomia para conduzir seus processos e práticas educativos. O sistema de governança está representado pelos conselheiros executivos e acadêmicos, professores, funcionários, alunos, egressos e membros da sociedade civil organizada.

A pós-graduação na UofA busca uma perfeita integração entre ensino-pesquisa-serviço. A internacionalização desempenha um importante papel nesse processo, uma vez que a diversidade cultural promove um ambiente acadêmico único de aprendizagem. De acordo com o conselho da Faculdade de Pós-graduação e Pesquisa (Faculty of Graduate Studies and Research- FGSR, 2017a), a UofA tem atualmente 7.600 alunos de pós-graduação, sendo que 34\% são estrangeiros.

A Faculdade de Educação da universidade está dividida em sete departamentos: Estudos de Política Educacional; Psicologia Educacional; Estudos Educacionais; Educação em Ciências da Saúde; Estudos de Biblioteca e Informação e Educação Secundária. Os cursos oferecidos são o Mestrado em Educação (MEd), Doutorado em Educação (EdD), Doutorado em Filosofia (PhD) e Certificado pós-bacharelado (PBC).

No tocante ao acompanhamento das atividades desenvolvidas na pós-graduação, os documentos investigados (University of Alberta, 2012; 2017b) reafirmam que a avaliação por pares é fundamental para zelar pela aprendizagem entre aluno-professor e aluno-aluno. De acordo com o GFC e o Conselho de Governadores, em consonância 
com a Post-Secondary Learning ACT (Alberta, 2003), este tipo de avaliação permite vislumbrar resultados, tanto numa perspectiva somativa, quanto formativa. Ou seja, visa recompensar o desempenho e ao mesmo tempo fornecer feedback.

Os critérios para a avaliação dos membros da equipe da Instituição são discriminados no Manual de Referência (University of Alberta, 2017b) que é utilizado pelos comitês de avaliação de cada faculdade, chefes de departamentos, diretores e demais administradores. Para a área de educação, o Comitê de Avaliação do Corpo Docente (University of Alberta, 2012) tem como foco as atividades de ensino, pesquisa e serviço, distribuídas da seguinte forma: participação em programas de ensino diversificados; participação em pesquisas envolvendo a preparação de trabalho crítico e reflexivo, com vistas à disseminação dos resultados; participação na governança institucional; prestação de serviços junto à comunidade e divulgação do conhecimento produzido.

Apesar do desempenho ser realçado quanto ao corpo docente, a natureza das atividades desenvolvidas deve se voltar para a disseminação da pesquisa na comunidade (University of Alberta, 2017a). A avaliação do professor é realizada anualmente, conduzida pelo representante do departamento, que deve elencar o maior número possível de informações para serem encaminhadas ao comitê da área (University of Alberta, 2012).

Quanto à avaliação discente (Alberta, 2003), a instituição destaca que seus alunos, independente da área, programa ou curso, devem participar efetivamente da governança da instituição, além de primarem pelo bom desempenho acadêmico. Para tanto, o aluno deve buscar o envolvimento nas diversas atividades do curso, debates em sala de aula, participação em eventos e inserção social na comunidade através de projetos e estudos. Na pós-graduação, o acompanhamento das atividades discentes é realizado pelos professores e pelo supervisor da dissertação ou tese. A FGSR (2017) ressalta que o aluno deve fornecer um relatório anual das atividades desenvolvidas no curso, como forma de registro da sua avaliação.

Os documentos investigados apontam uma maior autonomia entre o aluno e seu supervisor, inclusive quanto ao tempo integralizado para concluir o curso, sendo 6 anos para o doutorado e 4 para o mestrado. Contudo, a avaliação destaca aspectos ligados ao desempenho e ao mérito, especialmente quando se trata de discentes que são beneficiados com algum tipo suporte financeiro. De acordo com o FGSR (2017), as bolsas ou prêmios ofertados provêm do governo canadense ou provincial e da sociedade civil. Neste último caso, os recursos se originam principalmente de empresas que têm interesse em uma determinada pesquisa.

A relação cada vez mais estreita entre universidade e setor privado incita uma mudança na cultura acadêmica que, segundo Schugurensky e Naidorf (2004, p. 1001), tem "um novo modelo (muito mais profundo e global), caracterizado pela intensificação 
da transferência de tecnologia das universidades para a empresa e a comercialização do trabalho acadêmico". Na área de educação, as bolsas estão vinculadas a duas modalidades distintas de prestação de serviços: assistente de ensino de graduação ou assistente de pesquisa de pós-graduação (Alberta, 2003).

Quanto à pesquisa, a área de educação valoriza a pesquisa básica e aplicada e menciona a necessidade de uma avaliação qualitativa e quantitativa. Os professores devem atender a componentes diferentes que envolvam a contribuição com pesquisas acadêmicas, publicação de artigos em periódicos, capítulos de livro, edição e revisão, entre outros (University of Alberta, 2012). De modo geral, os documentos também apontaram uma relação mais harmoniosa entre a prática do ensino e da pesquisa. Isso talvez esteja relacionado ao fato de que a pós-graduação da UofA seja conduzida com maior autonomia tanto na perspectiva docente quanto discente, o que possibilita uma análise mais atenta às especificidades.

\section{A pós-graduação em educação: aproximações e distanciamentos}

O estudo sobre a pós-graduação no Brasil e no Canadá permitiu vislumbrar que, apesar das diferenças demográficas, históricas e sócioculturais, o modus operandi pelo qual a pós-graduação é sistematizada e a cultura acadêmica é modificada perpassa pela lógica da avaliação e da performance (Dias Sobrinho, 2003; Schugurensky \& Naidorf, 2004). De modo a contribuir com uma análise mais dinâmica, as informações levantadas foram tecidas com base nas seguintes categorias: organicidade da pós-graduação, avaliação do corpo docente e discente, produção intelectual e inserção social.

A organicidade da pós-graduação no Brasil e no Canadá evidencia algumas diferenças quanto à gestão e à autonomia. A política brasileira de pós-graduação integra um sistema mais centralizado, representado principalmente pela figura da Capes enquanto agência responsável pelo SNPG. A avaliação dos cursos segue os critérios padronizados constituintes nas fichas de cada área, o que sugere uma autonomia menor por parte da gestão dos programas, mesmo que a avaliação seja realizada pela perspectiva dos pares (Brasil, 2010).

No Brasil, apesar de cada IES estabelecer alguns critérios quanto ao ingresso nos cursos, número de disciplinas a serem cumpridas ou projetos de pesquisa, cabe ao programa atender às exigências da política externa de avaliação. Desse modo, as especificidades das áreas e das instituições ficam comprometidas e o nível de participação de docentes e discentes no processo é bastante limitado. Para Bianchetti, Valle e Pereira (2015) a lógica da avaliação, como se tem hoje, induz ao ranqueamento e à competição entre os programas e os próprios pares e impede a condução de uma análise mais pormenorizada de cada IES. 
No caso da pós-graduação canadense, como cada IES conduz sua avaliação, apesar de ter o amparo da lei provincial (Alberta, 2003), é possível perceber um modelo mais heterogêneo. Jones e Oleksiyenko (2011) explicam que, como não há a figura do Ministro Federal de Educação ou uma política para a educação superior, a avaliação se mostra mais descentralizada. Além do mais, identifica-se um envolvimento maior da comunidade acadêmica nas ações institucionais, especialmente no que se refere à avaliação, dado o modelo de governança educacional adotado pela University of Alberta.

A dinâmica da pós-graduação nestes países é um reflexo da mudança estrutural das políticas de educação superior. Ao contrário do Canada, a educação superior no contexto brasileiro é mais centralizada, cuja administração e regulamentação fica a cargo do MEC. Apesar das diferenças, a influência da globalização e das parcerias público-privadas podem ser claramente identificadas, sendo que, no Brasil, houve uma massificação das instituições privadas, promovendo a adoção de políticas incrementais de avaliação (Boaventura, 2009). No Canadá, o apoio irrestrito da indústria à pesquisa e às universidades públicas (Jones \& Oleksiyenko, 2011) incide na expectativa que se tem sobre os resultados e a formação acadêmica.

Em se tratando da avaliação do corpo docente e discente, há um consenso em ambos os contextos quanto à qualidade do ensino ofertado na pós-graduação e ao desempenho dos seus agentes. Nos documentos analisados, Brasil e Canadá ressaltam a necessidade de atender a critérios relacionados à criatividade, desempenho, produtividade, competitividade e disseminação da pesquisa (University of Alberta, 2012; Brasil, 2010).

Contudo, o modelo de avaliação preconizado na University of Alberta sugere maior autonomia tanto para docentes quanto para os discentes conduzirem suas pesquisas, o que permite inferir que há uma cobrança menor quanto ao aspecto da produtividade. Isso porque os documentos da área de educação elucidam que a avaliação deve considerar a natureza de cada atividade envolvida (University of Alberta, 2012; 2017a).

No Canadá, a avaliação docente leva em consideração todas as atividades desenvolvidas, sejam elas de ensino, pesquisa ou serviço. Mesmo que um professor não tenha um número significativo de publicações num determinado período, será observada a sua contribuição para a sociedade na forma de projetos, parcerias, consultorias, artigos entre outros. No caso dos discentes, a avaliação também evidencia a autonomia no cumprimento dos requisitos acadêmicos (University of Alberta, 2017a; 2017b), mas deixa explícito uma cobrança maior no caso dos alunos bolsistas que, além de cumprirem todos os requisitos do curso, devem prestar serviços à faculdade (FGSR, 2017). Ademais, percebe-se que os critérios de avaliação procuram manter um equilíbrio entre o aspecto quantitativo e o qualitativo, especialmente pelo modo como a avaliação por pares é conduzida na IES, permitindo um olhar mais profundo sobre todas as atividades executadas pelo corpo acadêmico (University of Alberta, 2012). 
No Brasil, apesar da avaliação ser realizada dentro da lógica dos pares, a centralidade na condução dessa política prejudica a adoção de critérios mais qualitativos tendo em vista o tamanho da área de educação e o número limitado de avaliadores para dar conta de todas as pesquisas produzidas, conforme apontam Barata (2017) e o próprio documento de área (Brasil, 2016).

Desse modo, a produção intelectual e a inserção social no contexto brasileiro, enfrentam um desafio maior para se reafirmarem. Percebe-se que a política de avaliação em educação enfatiza maior pressão por produtividade para os pesquisadores brasileiros, já que seus critérios se voltam, principalmente, para o número de publicações (Brasil, 2016). Já no Canadá, com a autonomia concedida para os professores e os alunos, esta pressão tende a ser minimizada, uma vez que a política adotada neste país também procura estimular de modo mais dinâmico, a inserção social da pesquisa (University of Alberta, 2012).

Entretanto, em ambos os contextos, a máxima da globalização pode trazer danos profundos para a educação quando vem revestida de um apelo competitivo. Apesar da pós-graduação nesses contextos ser gestada por modelos estruturais tão diversos, nota-se a máxima da mudança na cultura acadêmica, conforme explicam Schugurensky e Naidorf (2004). De um lado, o Brasil tem conduzido suas políticas de educação superior com um enfoque produtivista e quantitativista. Por outro lado, a maior relação entre a pesquisa e o serviço na UofA se mostra alinhada com a nova forma de pensar a educação superior, cada vez mais empreendedora e globalizada, uma vez que as doações do setor privado para a IES revelam um interesse de caráter capitalista.

Todos esses fatores afetam o posicionamento crítico e a prática reflexiva da pesquisa. A respeito disso, Nóvoa (2014) ressalta que é preciso "alargar o espectro das nossas maneiras de pensar e de falar sobre educação. Aprofundar o nosso compromisso com a inclusão, a educação e a cultura. É para isso que serve a investigação educacional” (p. 20). Bourdieu (2004) contribui com esta reflexão, ao afirmar que reduzir a área de educação a um instrumento de produtividade e de aliança econômica implica aceitar a transformação da alma do intelectual passando de um cientista engajado a um capitalista cada vez mais competitivo.

\section{Considerações finais}

O presente trabalho buscou identificar as aproximações e os distanciamentos na pós-graduação em educação no Brasil e no Canadá. Em geral, a atual política do nível stricto sensu em ambos os países está alinhada com as transformações vivenciadas no campo educacional nas últimas décadas, como resultado da globalização e de uma nova cultura acadêmica, com vistas à ampliação das parcerias público-privadas e a adoção de práticas empreendedoras. 
A lógica vigente ressalta aspectos ligados à governança e à regulação que são conduzidos de modos diferentes em cada contexto, mas reafirmam a mudança estrutural pela qual a pesquisa e a ciência têm passado. As aproximações da pós-graduação no Brasil e no Canadá esbarram no aspecto da avaliação e da mercadorização do conhecimento. Contudo, a centralidade da política de avaliação vivenciada pela área de educação confere ao contexto brasileiro a adoção de critérios mais quantitativos e maior pressão sobre docentes e discentes para produzir e publicar.

O que distancia a política adotada nesses modelos concerne à autonomia e ao tempo para maturação da pesquisa que, no Canadá, proporciona maior liberdade aos docentes e discentes para conduzirem seus estudos e, em razão disso, conseguem promover maior e melhor aproximação entre pesquisa e prática social. Entretanto, é importante destacar que os alunos bolsistas da UofA estão sujeitos a maiores cobranças no processo de avaliação.

É preciso reconhecer que a formação dos intelectuais em educação, especialmente no Brasil, enfatiza a competitividade e o ranqueamento, o que acaba promovendo um alijamento entre a pesquisa e a inserção social. No Canadá, a atenção se volta ao modo como a pesquisa tem sido conduzida, colocada cada vez mais a serviço do mercado, dado à estreita relação entre universidade e setor privado. Os apontamentos tecidos não sugerem uma postura contra a atual política da pós-graduação no Brasil ou no Canadá, mas visa compreender os desafios, impasses e perspectivas enfrentados, sobretudo quanto à adoção de critérios quantitativos que podem comprometer a avaliação da qualidade e do real impacto das pesquisas em educação para o desenvolvimento social.

\section{Notas:}

'Informações divulgadas pela Faculdade de Pós-graduação e Pesquisa (Faculty of Graduate Studies and Research - FGSR) do ranking QS World University 2016-2017. Recuperado de https:// www.ualberta.ca/graduate-studies

${ }^{2}$ Recuperado de https://geocapes.capes.gov.br/geocapes/

\section{Referências}

Aberta, Province of (2003). Post-secondary learning Act. Province of Alberta: Alberta Queen's Printer, Edmonton.

Barata, R. C. B. (2017). Impasses e perspectivas da avaliação. Fortaleza: Seminário.

Bianchetti, L., Valle, I. R., \& Pereira, G. R. M. (2015). O fim dos intelectuais acadêmicos? Induções da Capes e desafios às associações científicas. Campinas: Autores Associados.

Boaventura, E. M. (2009). Newton Sucupira, a pós-graduação e a universidade. EDUFBA, 143-153. Disponível em http://books.scielo.org/id/4r/pdf/boaventura-9788523208936-09.pdf

Bourdieu, P. (2004). Os usos sociais da ciência: por uma sociologia do campo científico. São Paulo: UNESP. 
Brasil. (2016). Coordenação de Aperfeiçoamento de Pessoal de Nível Superior (CAPES). Documento de área 2016. Disponível em http://www.capes.gov.br/images/documentos/Documentos_de_ area_2017/Educa\%C3\%A7\%C3\%A3o.pdf

Brasil. (2010). Coordenação de Aperfeiçoamento de Pessoal de Nível Superior. Plano Nacional de PósGraduação (PNPG) 2011-2020 (Vol. 01). Disponível em http://www.capes.gov.br/sobre-a-capes/ plano-nacional-de-pos-graduacao

Dias Sobrinho, J. (2003). Avaliação: políticas educacionais e reformas da Educação Superior. São Paulo: Cortez.

Faculty of Graduate Studies and Research (FGSR). (2017). Prospective students and current students. Disponível em https://www.ualberta.ca/graduate-studies/about

Franco, M. C. (2000). Quando nós somos o outro: questões teórico-metodológicas sobre estudos comparados. Educação \& Sociedade, 21 (72), 197-230.

Jones, G. A., \& Oleksiyenko, A. (2011). The internationalization of Canadian university research: a global higher education matrix analysis of multi-level governance. Higher Education, 61, 41-57.

Mailhot, C., Pelletier, P., \& Shaeffer, V. (2007). La valorization de la recherche: une nouvelle mission pour I'université? Canadian Journal of Higher Education, 37 (1), 45-65.

Moreira, A. F. (2009). A cultura da performatividade e a avaliação da pós-graduação em educação no Brasil. Educação em Revista, 25(3), 23-42. Disponível em http://www.scielo.br/pdf/edur/v25n3/03. pdf

Nóvoa, A. (2014). Em busca da liberdade nas universidades: para que serve a investigação em Educação? Revista Lusófona de Educação, 28(28), 11-21. Disponível em http://www.scielo.br/pdf/ ep/v41n1/1517-9702-ep-41-1-0263.pdf

Oliveira, R. P., Amaral, N. C., \& Zaidan, S. (2015). Acompanhamento de meio termo SNPG. Brasília: Seminário.

Rhodes, R. A. W. (1996). The new governance: governing without government. Political Studies, 44, 652-667.

Schugurensky, D., Naidorf, J. (2004). Parceria universidade-empresa e mudanças na cultura acadêmica: análise comparativa dos casos da Argentina e do Canadá. Educação \& Sociedade, 25(88), 9971022.

Severino, A. J. (2011). A Avaliação no PNPG 2005-2010 e a política de pós-graduação no Brasil. In N. S. C. Ferreira (Org.). Políticas públicas e gestão da educação: polêmicas, fundamentos e análises (pp. 49-72.). Brasília: Liber Livro.

Shiroma, E. O., Campos, R. F., \& Garcia, R. M. C. (2005). Decifrar textos para compreender a política: subsídios teóricos-metodológicos para a análise de documentos. Perspectiva, 23(2), 427-446. Disponível em https://periodicos.ufsc.br/index.php/perspectiva/article/view/9769/8999

University of Alberta. (2012). Faculty of Education Faculty Evaluation Committee. Criteria for the evaluation of academic staff, 1-12. Disponível em https://educadmin.ualberta.ca/sites/educadmin.ualberta.ca/ files/Criteria\%20for\%20Evaluation\%20of\%20Academic\%20Staff\%202015\%20Update.pdf 
University of Alberta. (2017a). Faculty agreement. Disponível em https://educadmin.ualberta.ca/content/ governance

University of Alberta. (2017b). Reference manual for faculty evaluation committees. Disponível em https:// educadmin.ualberta.ca/content/governance

Viczko, M. \& Tascón, C. I. (2016). Performing internationalization of higher education in Canadian national policy. Canadian Journal of Higher Education. Revue canadienne d'enseignement supérieur. 46(2), $1-18$.

Zheng, J. (2010). Neoliberal globalization, higher education policies and international student flows: an exploratory case study of chinese graduate student flows to Canada. Journal of Alternative Perspectives in the Social Sciences 2(1), 216-244.

Isabela Cristina Marins Braga

É doutoranda em Educação na Universidade Católica de Brasília/Brasil. Foi bolsista da Capes na modalidade Doutorado Sanduíche no Exterior, no período de agosto/2017 a fevereiro/2018, realizado na University of Alberta, Canadá. É Professora da educação superior na Faculdade Cnec Unaí/Unaí-

MG e na Faculdade de Ciências e Tecnologia de Unaí (FACTU) Unaí-MG.

E-mail: isabelaunai@gmail.com ORCID: http://orcid.org/0000-0001-9709-1903

Ranilce Guimarães-Iosif Adjunct Assistant Professor at the Department of Educational Policy Studies, University of Alberta, Canadá. Especialista em Educação da Secretaria de Educação do Distrito Federal, Brasília, Brasil.

E-mail: ranilceguimaraes@yahoo.com.br ORCID: http://orcid.org/0000-0002-5146-293X

Lynette Shultz Associate Dean (International) in the Faculty of Education and Professor in the Department of Educational Policy Studies at the University of Alberta in

Canada.

E-mail: Ishultz@ualberta.ca ORCID: http://orcid.org/ 0000-0002-3875-6992

Correspondência Isabela Cristina Marins Braga Rua Afonso Pena, 339, Centro - Unaí-MG, CEP: 38610-000 - Brasil

Data de submissão: Novembro 2017 Data de avaliação: Março 2018 Data de publicação: Setembro 2018 